\title{
Image of the month: Ventricular myxoma mimicking hypertrophic cardiomyopathy
}

\author{
Authors: Avinash Mani, ${ }^{\mathrm{A}}$ Arun Gopalakrishnan, ${ }^{\mathrm{B}}$ Anoop Ayyappan ${ }^{\mathrm{C}}$ and Ajitkumar Valaparambil ${ }^{\mathrm{D}}$
}

A 5-year-old boy with an incidentally detected cardiac murmur was referred for evaluation. Tall $\mathrm{R}$ waves were noted in the electrocardiogram in leads V3 and V4. Transthoracic echocardiography suggested asymmetric septal hypertrophy with diffuse thickening of the inter-ventricular septum with normal thickness of the posterior left ventricular wall. Upon closer interrogation, a masquerading sessile cardiac mass was identified adherent to the left ventricular side of the interventricular septum which appeared to contract with each cardiac cycle, mimicking hypertrophic cardiomyopathy.

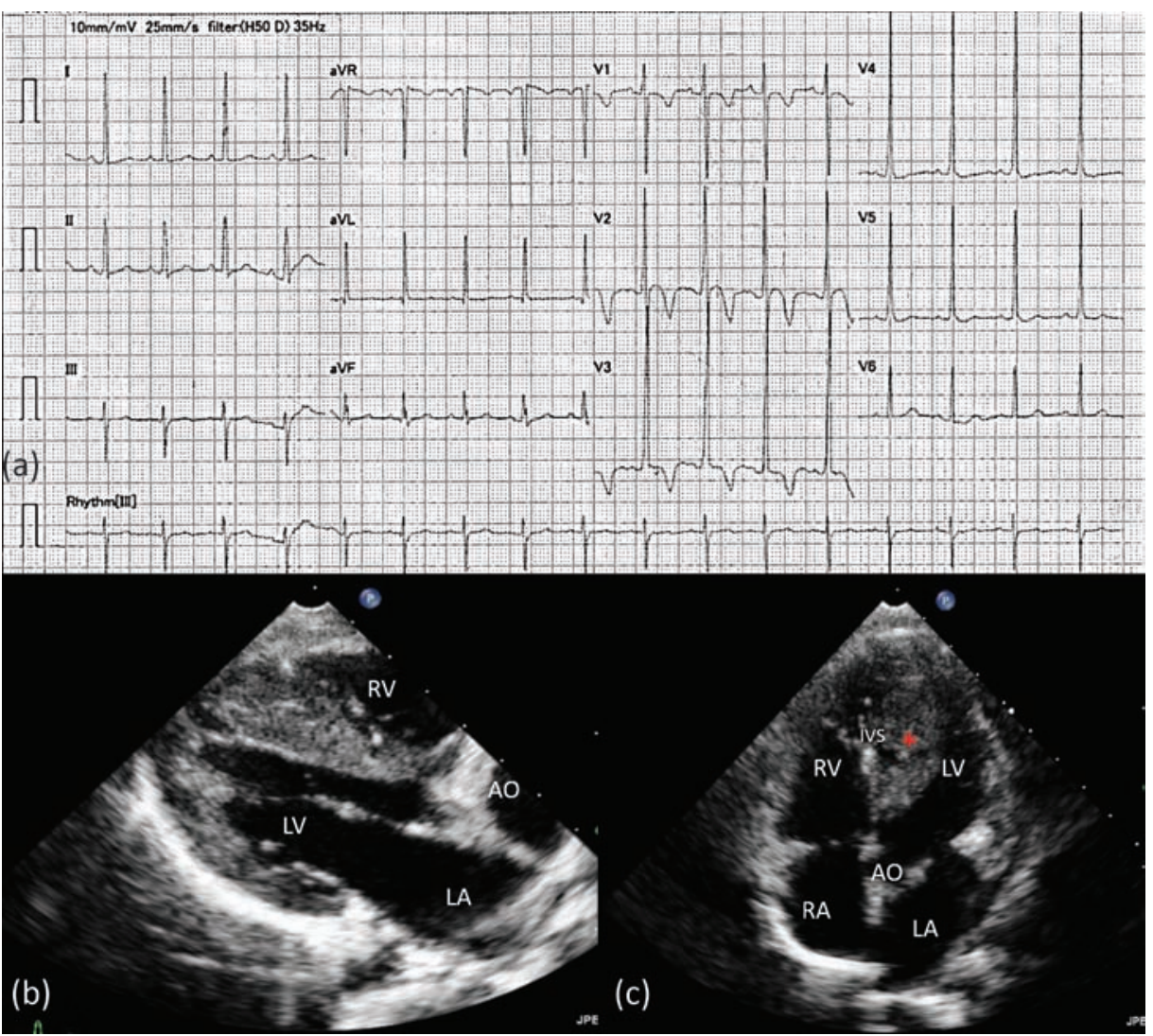

Fig 1. Electrocardiogram and transthoracic echocardiograms. Fig 1a is the 12-lead electrocardiogram of the patient showing sinus rhythm, normal PR interval and QRS axis but tall $\mathrm{R}$ waves in $\mathrm{V} 3, \mathrm{~V} 4$. Borderline LVH is evident. Fig $1 \mathrm{~b}$ is the transthoracic echocardiographic still frame in diastole from the parasternal long-axis projection suggesting asymmetric septal hypertrophy. The ventricular myxoma (red asterisk) is inconspicuous in this view. Fig $1 \mathrm{c}$ is the transthoracic echocardiographic still frame in diastole from the apical five-chamber projection showing a small plane of demarcation between the ventricular myxoma and the inter-ventricular septum differentiating the tumour from asymmetric ventricular hypertrophy. $\mathrm{AO}=$ aorta; $\mathrm{IVS}=$ inter-ventricular septum; $\mathrm{LA}=$ left atrium; $\mathrm{LV}=$ left ventricle; $\mathrm{RA}=$ right atrium; $\mathrm{RV}=$ right ventricle.

Authors: ${ }^{\text {A }}$ senior resident, Sree Chitra Tirunal Institute for Medical Sciences and Technology, Thiruvananthapuram, India; ${ }^{B}$ assistant professor, Sree Chitra Tirunal Institute for Medical Sciences and Technology, Thiruvananthapuram, India; ' assistant professor, Sree Chitra Tirunal Institute for Medical Sciences and Technology, Thiruvananthapuram, India; ${ }^{D}$ senior professor and head, Sree Chitra Tirunal Institute for Medical Sciences and Technology, Thiruvananthapuram, India; Sree Chitra Tirunal Institute for Medical Sciences and Technology, Thiruvananthapuram, India
KEYWORDS: Cardiac tumour, hypertrophic cardiomyopathy, asymmetric septal hypertrophy, echocardiography, myxoma

A 5-year-old boy was evaluated for an incidentally detected cardiac murmur during a dental check-up. 12-lead electrocardiogram showed tall R waves in V3 and V4 (Fig 1a). Transthoracic echocardiography suggested asymmetric septal hypertrophy with diffuse thickening of the inter-ventricular 
Fig 2. Cardiac magnetic resonance imaging (MRI) images of the patient. Fig $2 \mathrm{a}$ is the axial section showing the cardiac mass (red asterisk) with stalks of attachment (arrows) mimicking septal hypertrophy. Fig $2 \mathrm{~b}$ is the T1weighted short axis MRI still image showing that the mass is isointense with the myocardium. Fig $2 \mathrm{c}$ shows that the mass is hyperintense on T2-weighted imaging. Fig $2 d$ is the late gadolinium sequence of the MRI showing peripheral tumour enhancement (arrows) and mild central enhancement.

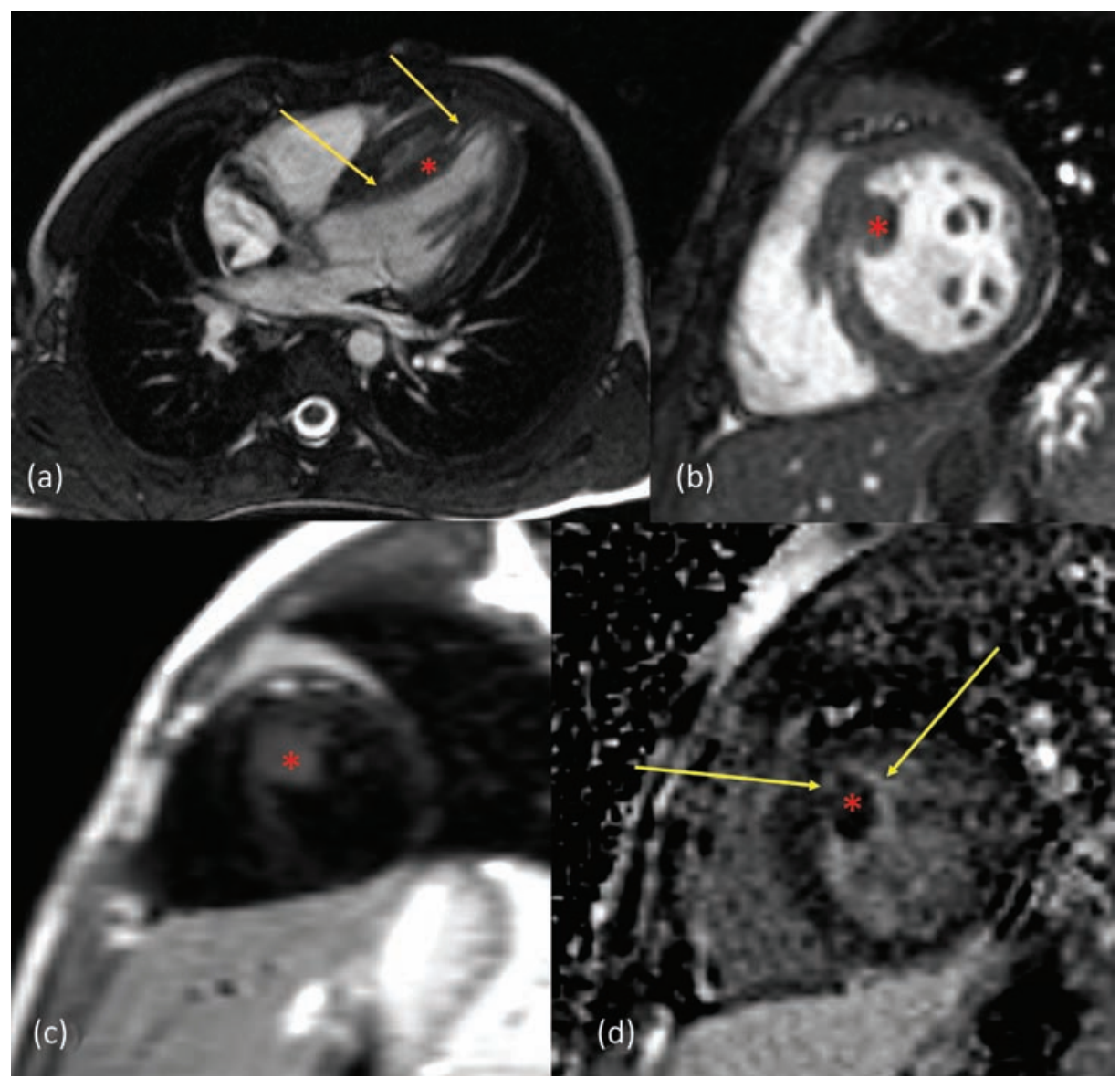

septum with normal thickness of the posterior left ventricular wall (Fig 1b-c). Upon closer interrogation, a masquerading sessile cardiac mass was identified adherent to the left ventricular side of the inter-ventricular septum which appeared to contract with each cardiac cycle. A clear plane of demarcation was identified from the inter-ventricular septum. The mass showed multiple short stalks of attachment to the septum at the apex and subaortic region. There was no inflow or outflow tract obstruction. Cardiac magnetic resonance imaging confirmed the exophytic lesion in the left ventricle, isointense on T1-imaging and mildly hyperintense on T2-weighted imaging (Fig 2). Delayed gadolinium enhancement sequences showed peripheral enhancement with gradual central enhancement suggesting a myxoma rather than a rhabdomyoma. The child was offered high-risk surgical excision after explaining the risks of incomplete excision and possible need for left ventriculotomy. However the parents preferred close medical follow-up, after explaining the risk of embolisation. There was no change in size or mobility of the mass till last follow-up at 6 months and the child remains asymptomatic.

The relevance of meticulous evaluation cannot be overemphasised during clinical examination and echocardiography. Sessile left ventricular myxomas are unusual and can pose diagnostic challenges, as in the index case. The risks of incomplete excision and possible tumour recurrence need to be weighed against the risk of embolisation with medical management.

Address for correspondence: Dr Arun Gopalakrishnan, Department of Cardiology, Sree Chitra Tirunal Institute for Medical Sciences and Technology, Thiruvananthapuram, Kerala 695011, India.

Email: arungopalakrishnan99@gmail.com 